\title{
Optimization of complex conservative treatment to patients of advanced and old age with atherosclerosis obliterans of lower limb arteries Stage II
}

\begin{abstract}
The article analyzes the results of treatment of 139 patients with chronic lower limb ischemia stage II in three parallel groups. The first group $(n=57)$ received standard conservative therapy in combination with ozone therapy; when this group was divided into two subgroups: Subgroup 1a patients $(\mathrm{n}=28)$ was performed intravenous ozonized saline (ODF), 1b subgroups $(\mathrm{n}=29)$ - greater autogemoozonoterapiyu (BAP). Patients of the second group $(\mathrm{n}=62)$ underwent a comprehensive treatment comprising medical ozone in addition to gravitational therapy (ADT). In this group as two subgroups were distinguished: 2 a subgroup $(\mathrm{n}=31)$ - patients receiving standard medical therapy in combination with HT and ODF, $2 \mathrm{~b}$ subgroup $(\mathrm{n}=31)$ - in combination with BAP and HT. The third group, control $(n=20)$ included patients receiving only standard medical therapy. The highest efficiency is noted in subgroup 2a. There was a statistically significant increase in pain-free walking distance by $200 \%$, ankle-brachial index $47 \%$.In this same subgroup seen most pronounced positive dynamics of indicators of the quality of life of patients, which was confirmed by the most efficient correction scales questionnaire SF-36: physical component index increased by $32 \%$, index of mental health $-29 \%$

Combined application of ozonated saline and gravitational therapy in patients with chronic lower extremity ischemia II step facilitates a significant increase in pain-free walking distance and ankle-brachial index and effectively improves the quality of life of patients.
\end{abstract}

Keywords: atherosclerosis, ozone therapy, gravitational therapy
Volume 3 Issue 6 - 2018

\author{
Makarov IV, Lukashou AV \\ Professor, Head of the Department of Surgical Disease, Samara \\ State Medical University, Russia
}

Correspondence: Igor V Makarov, Doctor of Medical Sciences, Head of the Department of Surgical Disease, Samara State Medical University, Russia, Email makarav-samgmu@yandex.ru

Received: October 17, 2018 | Published: November 09, 2018

\section{Introduction}

In spite of the great achievements of vascular surgery treatment of atherosclerotic lesions of lower limb arteries, still remains an actual problem of modern medicine. The main feature of said pathology is steadily progressive course. ${ }^{1-5}$

At the present stage nonmedicamental conservative therapies obliterating atherosclerosis lower limb arteries steadily gaining several positions and may even be more effective compared with surgery, especially in patients with II stage disease. ${ }^{6-10}$ The purpose of this work was to improve the results of complex treatment of patients with stage II atherosclerosis obliterating through the use of non-pharmacological exposure methods combining medical ozone and gravitational therapy.

\section{Materials and methods}

It was a prospective controlled study randomiziovannoe in three parallel groups in 139 patients with atherosclerosis obliterans of lower limb arteries IIA - IIB stages were on a planned in-patient treatment in the general surgery department MSH "CST station Samara JSC" Russian Railways ". The average age of patients with obliterating atherosclerosis of the lower extremities stage II was $71,24 \pm 8,49$ years. The groups were statistically comparable.
All patients included in the study received standard medical therapy. Three groups of patients were allocated. Patients treated with standard medical therapy in combination with ozone therapy constituted the first (1) group (57 men, mean age $71.2 \pm 9.3$ years). The group was divided into two subgroups: subgroup 1a-28 people received intravenous administration of ozonated saline $1 \mathrm{~b}$ subgroup - 29 people - most autogemoozonoterapiyu. In the second (2) group (62 patients, mean age $71.4 \pm 7.9$ years) complex treatment include, in addition to conservative treatment and standard medical ozone therapy gravity. This group was also divided into two subgroups: subgroup $2 \mathrm{a}$ - 31 people - received conservative therapy in combination with ozonated saline and gravitational therapy subgroup $2 b-31$ patients - conservative therapy with great autogemoozonoterapiey and gravitational therapy. 3 patients group (20 patients, mean age $71,0 \pm 8,4$ years) was appointed only the standard conservative therapy. Treatment duration averaged $10,1 \pm 0,11$ days.

Standard medical therapy in all groups included medical and physiotherapy treatment. To prevent depletion of endogenous antioxidants and avoid side effects during ODF and used BAP concentration gas mixture not exceeding $20 \mathrm{mg} / \mathrm{l}$, starting from a minimum $(5-10 \mathrm{mg} / \mathrm{L})$ at the first two procedures, then passing on the therapeutic dosage. At the same time the introduction of ODF was conducted on a daily basis, and BAT - every other day. 
Patients $2 \mathrm{a}$ and $2 \mathrm{~b}$ further subgroups daily from the third treatment ODF and the second session BAT through 1 hour after their closure, gravitational therapy sessions carried (only 8-10 sessions). For optimum adaptation to the gravitational accelerations carried out in therapy started gently with a minimum therapeutic dose1,5 G(32 $\mathrm{rev} / \mathrm{min}$ ) for 5-10minutes. Subsequently the rotation was gradually increased up to $10-15$ minutes and the number of revolutions to 36$38 \mathrm{rev} / \mathrm{min}$ with the maximum overdrive level to stop2,5 G (Russian Patents: "Method for the treatment of lower extremity arterial occlusive atherosclerosis» № 2475275 of 08.08 .2011 and "method of complex treatment of lower extremity arterial occlusive diseases» № 2523390 from $02 / 12 / 2013 \mathrm{~g}$ ).

It was conducted to evaluate the clinical effectiveness of treatments used by the research changes painless walking distance and $\mathrm{ABI}$ conducted before and after treatment. In carrying out the gravity centrifuge rotation therapy in conditions we investigated the dynamics of the system and the segmental blood pressure, with subsequent calculation LPI. In addition, patients completed a questionnaire SF36 quality of life.

\section{Results}

In the study of pain-free walking distance immediately after treatment there was a statistically significant maximum increase its Wilcoxon test at $200 \%$ in the subgroup $2 \mathrm{a}(\mathrm{P}=0.00004)$, and $122 \%$ in the subgroup $2 \mathrm{~b}(\mathrm{p}=0.001)$ (Table 1$)$.

Before treatment, all patients observed a decrease in ABI median when compared with the results obtained in patients without clinical and instrumental signs of atherosclerosis. In all test groups conducted after treatment significantly increased the LPI, LPI but the maximum increase occurred in the subgroup $2 \mathrm{a}-$ by $47 \%(\mathrm{p}=0.00001)$ and $2 \mathrm{~b}$ $46 \%(\mathrm{p}=0.00001)($ Table 2$)$

Table I The distance of painless walking $(\mathrm{m})$ before and after treatment, IU (25-75 quartiles)

\begin{tabular}{|c|c|c|c|c|c|}
\hline \multirow{2}{*}{$\begin{array}{l}\text { Dates observed } \\
\text { denia }\end{array}$} & \multicolumn{2}{|l|}{ Group $I(N=57) S$} & \multicolumn{2}{|l|}{ Group $2(N=62)$} & \multirow{2}{*}{$\begin{array}{l}\text { Group } 3(N \\
=20)\end{array}$} \\
\hline & Subgroup Ia $(n=28)$ & Subgroup Ib $(n=29)$ & 2a subgroup $(n=3 I)$ & $2 b$ subgroup $(n=3 I)$ & \\
\hline Before treatment & $100(53,5-150,0)$ & $100(78,5-243,5)$ & $100(58,0-201,0)$ & $87(50,0-120,0)$ & $98(50,0-130,0)$ \\
\hline After treatment & $160(120,5-243,5)$ & $15 I(|| 7,5-250)$ & $300(200,0-384,0)$ & $194(150,0-310,0)$ & $145(90,0-228,0)$ \\
\hline$R$ & 0.094 & 0,057 & 0.00004 & 0,001 & 0.54 \\
\hline
\end{tabular}

Me-median, p-the difference between the indices before and after treatment.

Table 2 Changing LPI before and after treatment, Me (25-75 quartiles)

\begin{tabular}{|c|c|c|c|c|c|c|}
\hline \multirow{2}{*}{$\begin{array}{l}\text { Dates } \\
\text { observed denia }\end{array}$} & \multicolumn{2}{|l|}{ Group I $(\mathbf{N}=\mathbf{5 7})$} & \multicolumn{2}{|l|}{ Group $2(N=62)$} & \multirow{2}{*}{$\begin{array}{l}\text { Group } 3(\mathbf{N} \\
=20)\end{array}$} & \multirow{2}{*}{$\begin{array}{l}\text { Healthy } \\
\text { good-volz (N } \\
=17)\end{array}$} \\
\hline & $\begin{array}{l}\text { Subgroup Ia (n } \\
=28)\end{array}$ & $\begin{array}{l}\text { Subgroup Ib } \\
(n=29)\end{array}$ & 2 a subgroup $(n=3 I)$ & $\begin{array}{l}\text { 2b subgroup ( } n \\
=3 I \text { ) }\end{array}$ & & \\
\hline Before leche-tion & $0.5 \mathrm{I}(0,6 \mathrm{I}-0,66) *$ & $0.5(0,4 I-0.55)^{\wedge}$ & $0.5 \mathrm{I}(0,4 \mathrm{I}-0.58)^{\wedge}$ & $0.47(0,4 I-0,49) *$ & $0.48(0.43-0.50) *$ & \multirow[b]{2}{*}{ I.I6(I,II-I,20) } \\
\hline After treatment & $0.64(0,52-0.73)^{\wedge}$ & $0.6(0,52-0,68)^{\wedge}$ & $0.75(0,68-0,83) \infty \times \#^{\wedge}$ & $0.68(0,63-0.77) \#^{\wedge}$ & $0.52(0,55-0,49) *$ & \\
\hline
\end{tabular}

Me -median, p-statistically significant differences between the indices before and after treatment, ${ }^{*}$ - statistically significant difference from the group of indicators without clinical and instrumental symptoms of atherosclerosis, \#-statistically significant differences with the control group $3, \infty$ - statistically significant difference from subgroup $1 \mathrm{a}, \times$ - statistically significant differences with the subgroup $2 \mathrm{~b}$. In group 2 patients, the treatment in which included and gravitational therapy and medical ozone were monitored LPI scheme:

a. lying at rest, when placing the patient on a short radius centrifuge cradle action

b. during a session gravitational therapy, in the seventh minute rotation

c. immediately after stopping the centrifuge (Figure 1).

During the first two sessions of gravitational therapy changes in blood flow in the arteries of the lower extremities were not significant, due to the adaptive mode load (1,5 $\mathrm{G}$ for 10 minutes). Median LPI during adaptation session in the subgroup $2 \mathrm{a}$, where the patients were pretreated with ozonated saline increased by $24 \%(\mathrm{p}=0.04)$ from 0.58 to 0.72 and immediately after stopping decreased and did not differ significantly from the initial readings $(\mathrm{p}=0.09)$. In a subgroup of figure $2 \mathrm{~b}$ the seventh minute gravitational therapy increased by $18 \%(\mathrm{p}=0.05)$ from 0.55 to 0.65 , and immediately after stopping the centrifuge has not changed significantly $(\mathrm{p}=0.12)$ (Figure 1$)$.

When conducting multiple comparisons during the next sessions of gravitational therapy with nominal modes of rotation of 36$38 \mathrm{rpm}$, the monitoring of ABI showed more significant changes in the macrohemodynamics of the lower extremities. During the third session, a more pronounced increase in the median ABI was noted, with the most significant in subgroup $2 \mathrm{a}-$ by $74 \%(\mathrm{p}=0.00001)$ from 0.61 to 1.06 (during the hypergravity session ), and in subgroup $2 \mathrm{~b}$ by $65 \%(\mathrm{p}=0.002)$ from 0.57 to 0.94 (during the hypergravity session ). From session to session, there was a trend towards an increase in the median of the ABI indicator, which was measured before the session of gravitational therapy (Figure 1). 


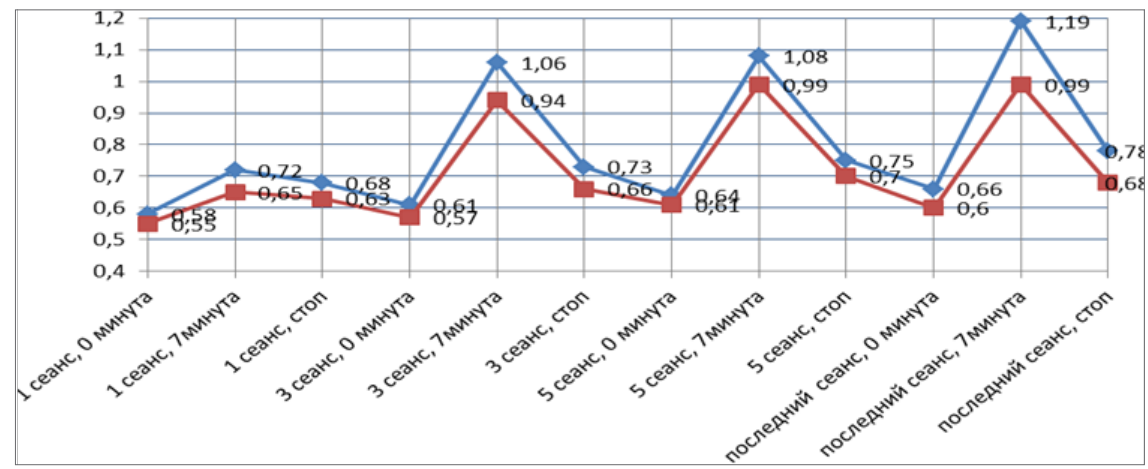

Figure I Changes in the ankle-brachial index during the sessions of gravitational therapy, Me (25-75 quartiles), P2A-2b $<0.05$.

Thus, before the course of treatment, all the examined patients had stage II atherosclerosis obliterans of the lower limb arteries, while the majority of patients were in stage IIB-107 people (77\%), II A in 32 patients $(23 \%)$. After the course of treatment, the calculation of the coefficient of association $\varphi$ was made. In the subgroup 2a, a statistically significant maximum effect was observed, where $\varphi=0.79$, with $\mathrm{p}=0.01$. The number of patients in this subgroup maximally reduced by $37 \%$, not only due to the transition stage in II B IIA, but in step I in $6 \%$ of patients.

For the analysis of the SF36 questionnaire before and after treatment, the 8 scales were grouped into two main integral indicators: physical and mental well-being. When studying before the start of treatment, both indicators differed significantly from the data of healthy volunteers $(p>0.05)$. After treatment in the subgroup $2 a$, the indicator of the physical component was statistically significant and maximally increased by $32 \%$ (at $p=0.001$ ), and in subgroup $2 b$ - by $16 \%$ (at $\mathrm{p}=0.01$ ). A similar trend was observed in the change in the index of mental health (an increase of $29 \%$ at $\mathrm{p}=0.009$ and $17 \%$ at $\mathrm{p}=0.02$, respectively ).

\section{Conclusion}

Considering the findings, it can be argued that the combined use of medical ozone (OFR or BAT) and gravitational therapy as part of complex treatment of patients with obliterating atherosclerosis has a pronounced positive effect on the course of the disease by stimulating the development of collateral peripheral blood flow and microcirculation. As a result of a comparative analysis, the highest efficiency was observed in the subgroup of patients receiving a combination of FEV and HT, where an increase in the distance of painless walking was noted by $200 \%$, and LEP - by $49 \%$.

The proposed method, including the combined use of OFR and HT, was easily tolerated by patients, was technically easy to implement and allowed to achieve good results in the treatment of patients with atherosclerosis obliterans of lower limb artery disease stage II, which allows it to be recommended for widespread use in clinical practice.

\section{Acknowledgements}

None.

\section{Conflict of interest}

Author declares that there is no conflict of interest.

\section{References}

1. Kazantsev AV. Diagnosis of progressive course of atherosclerosis obliterans of lower limb arteries based on indicators of endothelial dysfunction. Medical Bulletin of Bashkortostan. 2011;1:28-31.

2. Pizova NV. Atherosclerotic lesion of the carotid arteries in young patients. Clinician. 2014;1:28.

3. Vinnik, Yu S. Quality of life in patients with obliterating atherosclerosis of the vessels of the lower extremities according to the questionnaire SF-36. Fundamental research. 2015;1-3:467-469.

4. Zhdanov VS. Epidemiological morphological studies of atherosclerosis. Cardiological Gazette. 2015;1:52-57.

5. Antozhskaya, Yu S. Lipid metabolism in patients with atherosclerosis, depending on the focal nature of the lesion and the severity of lower limb ischemia. Medicine. 2011;3:85-90.

6. Polyakov PI. Obliterating atherosclerosis of the lower extremities in the elderly. Bulletin of new medical technologies. 2013;1: 98-101.

7. Galkin RA. Gravitational therapy in the treatment of patients with obliterating diseases of the arteries of the lower extremities. Samara. 2006. $198 \mathrm{p}$.

8. Kotelnikov GP. Gravitational therapy. Medicine. 2003. 244 p.

9. Kostyuchenko AL. Efferent therapy. AL Kostyuchenko-SPb: Folio; 2003. $432 \mathrm{p}$.

10. EI Nazarov, VG Wongai, TA Deaf. Pharmacological profile of ozone in the procedures of large autohemotherapy and intravenous infusion of ozonized saline. Bulletin of physiotherapy and balneology; 2012:6-9. 